\title{
A novel immunodeficiency associated with hypomorphic RAG1 mutations and CMV infection
}

\author{
Jean-Pierre de Villartay, ${ }^{1,2}$ Annick Lim, ${ }^{3}$ Hamoud Al-Mousa, ${ }^{4}$ Sophie Dupont, ${ }^{5}$ \\ Julie Déchanet-Merville, ${ }^{6}$ Edith Coumau-Gatbois, ${ }^{4}$ Marie-Lise Gougeon, ${ }^{3}$ \\ Arnaud Lemainque, ${ }^{7}$ Céline Eidenschenk, ${ }^{8}$ Emmanuelle Jouanguy, ${ }^{8}$ Laurent Abel, ${ }^{8}$ \\ Jean-Laurent Casanova, ${ }^{4,8}$ Alain Fischer, ${ }^{1,4}$ and Françoise Le Deist ${ }^{1,2}$
}

\begin{abstract}
${ }^{1}$ Développement Normal et Pathologique du Système Immunitaire, INSERM U429, Paris, France. ${ }^{2}$ Centre d'étude des déficits immunitaires, Hôpital Necker-Enfants Malades, Paris, France. ${ }^{3}$ Unité d'immunité antivirale, de biothérapie et de vaccination, Institut Pasteur, Paris, France. ${ }^{4}$ Unité d'Immunologie-Hématologie Pédiatrique, Hôpital Necker-Enfants Malades, Paris, France. ${ }^{5}$ Département de Pédiatrie, Hôpital Sainte Justine, Montreal, Quebec, Canada. 6UMR CNRS 5164 CIRID Université Bordeaux, Bordeaux, France. ${ }^{7}$ Centre National de Génotypage, Evry, France.

${ }^{8}$ Laboratoire de Génétique Humaine des Maladies Infectieuses, Université de Paris René Descartes-INSERM U550, Paris, France.
\end{abstract}

\begin{abstract}
Amorphic mutations in the recombination activating genes $R A G 1$ and $R A G 2$ have been reported to cause $\mathrm{T}^{-} \mathrm{B}^{-}$ SCID, whereas hypomorphic mutations led to the expansion of a few autoimmune $T$ cell clones responsible for the Omenn syndrome phenotype. We report here a novel clinical and immunological phenotype associated with recessive $R A G 1$ hypomorphic mutations in 4 patients from 4 different families. The immunological phenotype consists of the oligoclonal expansion of TCR $\gamma \delta$ T cells combined with TCR $\alpha \beta$ T cell lymphopenia. The clinical phenotype consists of severe, disseminated CMV infection and autoimmune blood cell manifestations. Repertoire studies suggest that CMV infection, in the setting of this particular $T$ cell immunodeficiency, may have driven the TCR $\gamma \delta \mathrm{T}$ cell clonal expansion. This observation extends the range of clinical and immunological phenotypes associated with $R A G$ mutations, emphasizing the role of the genetic background and microbial environment in determining disease phenotype.
\end{abstract}

\section{Introduction}

SCID defines a group of genetic disorders characterized by profound defects in T cell differentiation (1). Ten SCID conditions have been identified, based on immunological, enzymatic, and molecular genetic analysis. Adenosine deaminase deficiency results in a profound impairment of T, B, and NK lymphocyte development (2). Defects in IL-dependent $\mathrm{T}$ and NK cell differentiation caused by mutations in the $\gamma c$ gene ( $\gamma c$ deficiency) or the $\gamma c$-associated Jak-3 kinase gene (JAK3 deficiency) result in the $\mathrm{T}^{-} \mathrm{B}^{+} \mathrm{NK}^{-}$SCID phenotype $(3,4)$. Deficiency of the $\alpha$ chain of the IL-7 receptor $(5)$ of CD45 $(6,7)$ and of either of the CD3 chains (CD3 $\delta$ and $\mathrm{CD} 3 \varepsilon$ ) accounts for the $\mathrm{T}^{-} \mathrm{B}^{+} \mathrm{NK}^{+}$type of $\operatorname{SCID}(8,9)$ phenotype. Finally, defective $\mathrm{V}(\mathrm{D}) \mathrm{J}$ recombination of the $\mathrm{T}$ and $\mathrm{B}$ cell receptors is responsible for the $\mathrm{T}^{-} \mathrm{B}^{-} \mathrm{NK}^{+}$form of SCID (10-12). Defects in recombination activating gene 1 (RAG1) and RAG2 or in the Artemis gene can disrupt V(D)J recombination, thereby blocking the differentiation of $\mathrm{T}$ and $\mathrm{B}$ cells. However, hypomorphic mutation of the RAG1/2 or Artemis genes may generate a different phenotype, characterized by residual $\mathrm{T} \pm \mathrm{B}$ cell differentiation. Various RAG1 and RAG2 mutations have been found to be associated with a spectrum of immunodeficiencies extending from $\mathrm{T}^{-} \mathrm{B}^{-} \mathrm{NK}^{+} \mathrm{SCID}$ to Omenn syndrome (OS) $(11,13)$. OS is characterized by the massive expansion of oligoclonal $\mathrm{T}$ lymphocytes $(11,13-15)$.

Nonstandard abbreviations used: BMT, bone marrow transplantation; OS, Omenn syndrome; P2, patient 2; PHA, phytohemagglutinin; RAG1, recombination activating gene 1 ; TCRVB, T cell receptor variable $\beta$ chain.

Conflict of interest: The authors have declared that no conflict of interest exists.

Citation for this article: J. Clin. Invest. 115:3291-3299 (2005).

doi:10.1172/JCI25178.
$\mathrm{TCR} \gamma \delta \mathrm{T}$ cells account for $1-10 \%$ of peripheral $\mathrm{T}$ cells in the blood, while they are more prominent in mucosal sites (16). They display a restricted repertoire, using a limited set of $\mathrm{V} \gamma$ and $\mathrm{V} \delta$ gene elements (16). There is now considerable evidence to suggest that TCR $\gamma \delta$ directly recognizes various microbiological products, such as nonpeptide phenyl pyrophosphates from Mycobacterium tuberculosis (17) and other microorganisms (18-21). Experimental data from mouse models strongly suggest that TCR $\gamma \delta$ T cells play a critical role in host defenses against a variety of infectious agents, including herpes simplex virus type 1 (22). In humans, the expansion of $\gamma \delta \mathrm{T}$ cell subsets has been documented in Mycobacterium infection (17) and in immunocompromised transplanted patients infected with the CMV (23-25).

In this study, we analyzed a novel inherited immunodeficiency caused by hypomorphic RAG1 mutations. This phenotype consists of TCR $\alpha \beta$ T cell lymphopenia combined with expansion of the $\mathrm{TCR} \gamma \delta \mathrm{T}$ cell population, severe CMV infection, and autoimmunity. We suggest that CMV infection triggers the expansion of TCR $\gamma \delta$ $\mathrm{T}$ cell clones in conditions of restricted $\mathrm{T}$ cell clonal expansion.

\section{Results}

Immunological characteristics. Four unrelated patients, each born to first-cousin parents, presented with severe, persistent CMV infection in the first year of life in the absence of evidence for prenatal CMV infection. In 3 cases, CMV infection was associated with autoimmune cytopenias (anemia in patient 2 [P2], P3, and P4 and neutropenia in P2). Autoimmunity was further demonstrated by the detection of autoantibodies against red blood cells in 3 patients (P2, P3, and P4) and anti-polymorphonuclear autoantibodies in $\mathrm{P} 2$. Anti-nuclear autoantibodies were also detected in P2 and P3. 


\section{Table 1}

Patients' immunological characteristics: blood lymphocyte subsets and proliferation studies

\begin{tabular}{|c|c|c|c|c|c|}
\hline & P1 & $\mathbf{P 2}$ & P3 & P4 & $\begin{array}{l}\text { Age-matched } \\
\text { control values }\end{array}$ \\
\hline Age at investigation (mo) & 3 & $12-19$ & 13 & 19 & \\
\hline Lymphocytes/ul & $1,100-1,400$ & $3,000-5,000$ & $4,000-5,000$ & 3,500 & $2,900-5,400$ \\
\hline Eosinophils/ul & $200-800$ & $60-100$ & $0-100$ & NE & $50-100$ \\
\hline \multicolumn{6}{|l|}{ Lymphocyte subsets (\%) } \\
\hline $\mathrm{CD}^{+}$ & 30 & $47-78$ & $68-81$ & 55 & $62-69$ \\
\hline $\mathrm{CD}^{+}$ & 5 & $5-21$ & $6-9$ & 4 & $30-40$ \\
\hline $\mathrm{CD}^{+}$ & 5 & $6-16$ & $4-10$ & 4 & $25-32$ \\
\hline CD19+ & 0 & $3-51$ & 4 & 12 & $21-28$ \\
\hline $\mathrm{CD}^{-} 6^{+} \mathrm{CD}^{-}$ & 34 & 13 & 13 & 38 & $4-15$ \\
\hline HLA class $\mathrm{II}^{+} / \mathrm{CD}^{+}$ & ND & 77 & 94 & 62 & $6-16$ \\
\hline \multicolumn{6}{|l|}{$\operatorname{TCR} \alpha \beta^{+} /(\%)$} \\
\hline TCR $\alpha \beta^{+} / \mathrm{CD}^{+}$ & $20-23$ & 20-33 & $14-19$ & 8 & $86-97$ \\
\hline $\mathrm{TCR} \alpha \beta^{+} / \mathrm{CD}^{+}$ & ND & 8 & 5 & 0.5 & $85-100$ \\
\hline $\mathrm{CD} 4 \mathrm{RA}+\mathrm{CD}^{+}+$ & ND & 1 & 1 & 1 & $66-88$ \\
\hline $\mathrm{CD} 45 \mathrm{RA}^{+} \mathrm{CD} 31^{+} / \mathrm{CD} 4+$ & ND & ND & ND & 1 & $60-80$ \\
\hline \multicolumn{6}{|l|}{ TCR $\gamma \delta^{+} /(\%)$} \\
\hline $\mathrm{TCR} \gamma \delta^{+} / \mathrm{CD}^{+}$ & $77-80$ & $64-80$ & $80-87$ & 82 & $2-13$ \\
\hline TCR $\gamma \delta^{+} / \mathrm{CD}^{+}{ }^{+}$ & ND & 82 & 86 & 72 & $0-15$ \\
\hline $\mathrm{CD} 45 \mathrm{R} 0+/ \mathrm{TCR} \gamma \delta^{+}$ & 93 & ND & 78 & ND & \\
\hline $\mathrm{CD}^{28^{+}} / \mathrm{TCR} \gamma \delta^{+}$ & 2 & ND & 4 & ND & \\
\hline $\mathrm{CD} 27^{+} / \mathrm{TCR} \gamma \delta^{+}$ & ND & ND & 5 & ND & \\
\hline \multicolumn{6}{|c|}{ Proliferation $\left(\Delta \times 10^{-3} \mathrm{cpm}\right)$} \\
\hline PHA & ND & 151 & 44 & ND & $>50$ \\
\hline Tetanus toxoid & ND & $95^{A}$ & $38^{A}$ & ND & $>15$ \\
\hline CMV & ND & $12^{A}$ & $4^{\mathrm{A}}$ & ND & $>15$ \\
\hline
\end{tabular}

NE, not evaluable; ND, not done. AAntigen-induced proliferation assays were positive in 1 test out of 4 in P2 and in 1 test out of 3 in P3.

Lymphocyte counts were normal in P2, P3, and P4 and low in P1 (Table 1). All lymphocyte subsets (T, B, NK) were detectable, and the counts for these subsets were close to normal values (except for P1). T cells were found to be of autologous origin, as shown by microsatellite analysis or by $\mathrm{X} / \mathrm{Y}$ chromosome FISH. All patients displayed a marked decrease in T cells expressing the $\alpha \beta$ form of the TCR (Table 1), with counts of these cells well below $1,000 / \mu 1$. This TCR $\alpha \beta^{+} \mathrm{T}$ cell deficiency persisted over several months (data not shown). The control of CMV infection by antiviral therapy had no effect on TCR $\alpha \beta$ T cell counts (data not shown). Both CD4 ${ }^{+}$ and $\mathrm{CD}^{+}{ }^{+} \mathrm{TCR} \alpha \beta \mathrm{T}$ cells were detected with a memory/activated phenotype (CD45RO/HLA class $\left.\mathrm{II}^{+}\right)$. The distribution of the TCR variable $\beta$ chain (TCRVB) was assessed by immunofluorescence using specific $\mathrm{mAb}$ against $\mathrm{CD} 4^{+} \mathrm{T}$ lymphocytes or by PCR analysis. All members of the TCRVB family tested were present, but some were in excess (such as VB14 in P2 and P4; VB20 in P2; and $\mathrm{VB} 3$ in P3) (Figure 1). We were unable to evaluate the distribution of TCRVB on CD8 ${ }^{+} \mathrm{T}$ lymphocytes because most CD8 T cell subsets expressed the $\gamma \delta$ TCR (see below). A second striking feature of these 4 patients was the overrepresentation of TCR $\gamma \delta^{+}$T cells (Table 1). We showed, with antibodies specific for the $\mathrm{V} \gamma$ and $\mathrm{V} \delta$ families and by PCR analysis, that these families were present in each patient but that their distribution differed between patients (Figure 2). We followed the distribution of these families over time in $\mathrm{P} 2$ and found it to be stable throughout a 4-month follow-up period. $\gamma \delta \mathrm{T}$ cells from P1 were mostly V 22 and $\mathrm{V} \delta 3 ; \gamma \delta \mathrm{T}$ cells from P2 were mostly V $\delta 1$ and $\mathrm{V} \delta 5 ; \gamma \delta \mathrm{T}$ cells from P3 were mostly V $\delta 1$; and $\gamma \delta$ $\mathrm{T}$ cells from $\mathrm{P} 4$ were mostly $\mathrm{V} \delta 2$. There was no obvious dominance of $V \gamma$ families (Figure 2). Of note, the physiological overrepresentation of $\mathrm{V} \gamma 9$ among blood $\gamma \delta \mathrm{T}$ cells was not found in these 4 patients. Analysis of the distribution of CDR3 length within the various $V \gamma$ and $V \delta$ $T$ cells from P2, P3, and P4 showed fewer peaks than observed in control $\gamma \delta$ T cells (Figure 2). Sequencing of CDR3 from peaks showed limited diversity, as shown in Table 2 for $V \delta 3$ and $\mathrm{V} \gamma 3 \mathrm{~T}$ cells clone for P4. This observation held true for all $V \delta$ and $V \gamma$ families, except for $V \gamma 9$, which did not display a Gaussian distribution in controls (Figure 2). In both patients tested (P1 and P3), TCR $\gamma \delta^{+} \mathrm{T}$ cells were shown to be

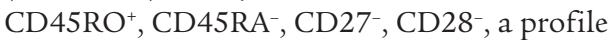
characteristic of late differentiated memory $\mathrm{T}$ cells (26) (Table 1). The general expansion of an oligoclonal TCR $\gamma \delta^{+} \mathrm{T}$ cell population with a TCRV $\delta$ and $-V \gamma$ usage different from that of the controls was therefore a feature common to these 4 patients. It was tested whether fresh or nonspecifically expanded $\gamma \delta \mathrm{T}$ cells from P1 and P3 could be activated by CMV-infected cells (25) either to proliferate, produce TNF- $\alpha$, or exert a cytolytic activity. Results were negative (data not shown). $\gamma \delta$ T cells could, however, proliferate in the presence of phytohemagglutinin (PHA) plus IL-2 (25).

In vitro, PHA-induced and antigen-induced proliferations in response to tetanus toxoid $(\mathrm{P} 2, \mathrm{P} 3)$ and CMV antigen (P2), respectively, were detected. Following exposure to antigen, only TCR $\alpha \beta \mathrm{CD}^{+}$ cells were found to proliferate (data not shown). The T cells of the patients therefore displayed no gross signaling defect. Serum Igs were detected in 3 cases (P2, P3, and P4). In contrast, P1 had profound panhypogammaglobulinemia. Serum IgE levels, tested in 3 cases, were low. Remarkably, when P2-P4 were tested, antibodies against CMV of both the IgM (in P2 and P4) and IgG (in P2 and P3) isotypes were detected. However, serum concentrations of antibodies against tetanus toxoid and poliovirus were very low in $\mathrm{P} 2$ following immunization (Table 3 ), indicating a functional B cell immunodeficiency.

Detection of homozygous RAG1 mutations. The occurrence of severe opportunistic infections associated with profoundly abnormal $\mathrm{T}$ cell subset distributions in these patients born to consanguineous parents was suggestive of an autosomal recessive inherited form of severe $\mathrm{T}$ cell immunodeficiency. The $\mathrm{NK}^{-}$SCID phenotype was excluded by the presence of NK cells, and enzyme assays excluded adenosine deaminase and purine nucleoside phosphorylase deficiencies. The normal membrane expression of both the TCR/CD3 complex and CD45 ruled out the possibility of a deficiency of these proteins. A T/B cell combined immunodeficiency caused by a defective $V(D) J$ TCR/BCR gene rearrangement was therefore considered, particularly as TCR heterogeneity was restricted in these patients, a characteristic of OS patients with leaky RAG1/2 mutations. Taking advantage of 2 informative consanguineous families (P2 and P3), a 

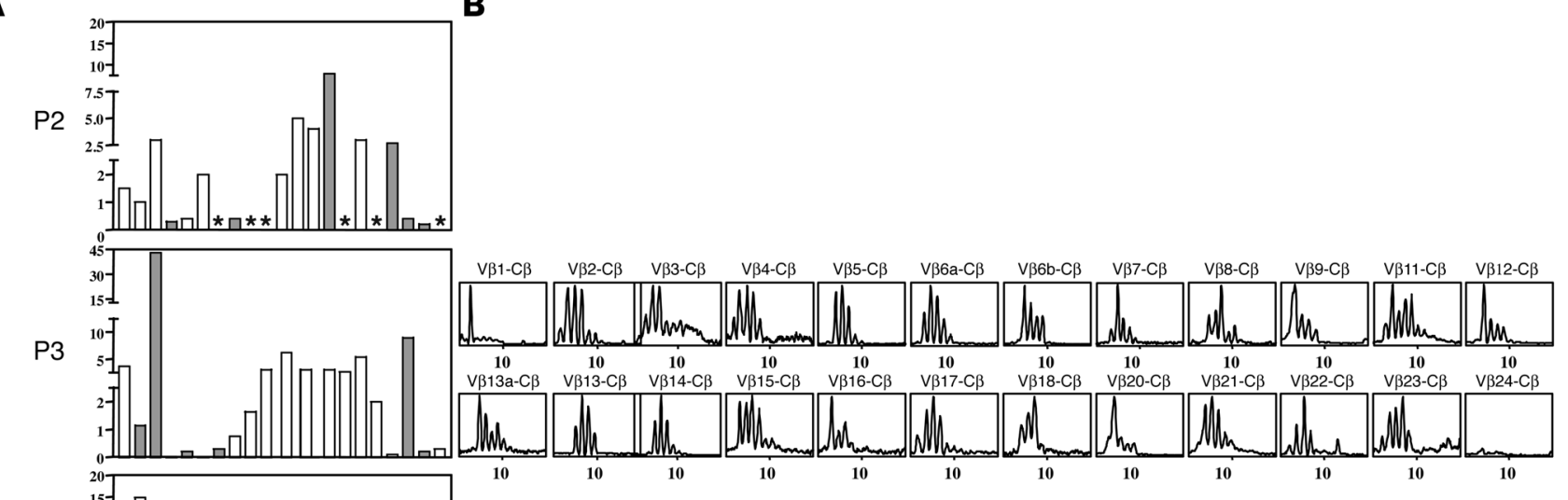

P4
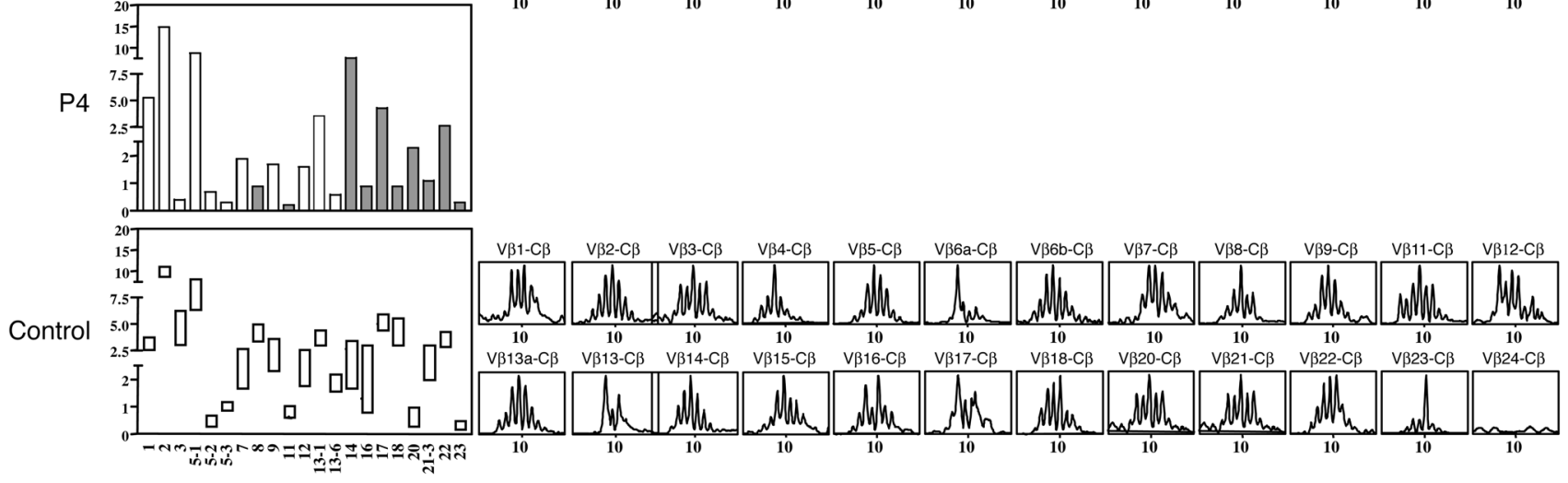

Figure 1

TCRVB usage by patients' T lymphocytes. (A) VB repertoire was determined either using available specific mAb in immunofluorescence assay in P2 and P4 or using real-time PCR analysis assay in P3. In this latter patient, similar results were obtained by PCR analysis and using available specific mAb in immunofluorescence assay (data not shown). The $x$ axis indicates VB families; the $y$ axis indicates relative frequency of usage (\%) within the TCR $\alpha \beta$ T cells. Controls values shown in rectangles represent mean \pm 2 SDs. Gray bars indicate abnormal VB usage. ${ }^{*}$ Not done. (B) Profiles of the fluorescent $\mathrm{V} \beta-\mathrm{C} \beta$ runoff products obtained with mononuclear cells. The $x$ axis indicates CDR3 length.

genome-wide scan was conducted by homozygosity mapping (27) using GENEHUNTER software (28). A total of 18 subjects belonging to 2 families were genotyped for a panel of 381 microsatellite markers. The Linkage Mapping Set MD-10 (Applied Biosystems) formed the core marker set for the scan. Linkage was found (multipoint lod scores between 2.85 and 3.00) to a chromosome $11 \mathrm{p}$ region spanning from D11S1338 (5.9 Mb) to D11S905 (40.9 Mb). The RAG1 gene is located in this chromosomal region (36.5 Mb).

Homozygous RAG1 gene mutations were identified in all 4 patients (Figure 3). The heterozygous status of both parents was confirmed in all cases. A homozygous del T631 mutation was identified in P1. This mutation, which results in a frameshift at $\mathrm{T} 173$ followed by a premature stop codon, has been reported in several T-B- SCID and OS patients as well $(11,13,29,30)$. It does not result in a complete loss of function, as translation may be reinitiated by use of an alternative ATG (M202), 3' to the frameshift deletion, resulting in a partially functional $\mathrm{N}$-terminally truncated RAG1 protein (31). The homozygous dinucleotide deletion del A368/A369 identified in P4, which has also been reported in previous studies, had similar consequences for RAG1 expression, with an $\mathrm{N}$-terminally truncated protein produced by translation initiation at M183 $(13,30,31)$. P2 carried a homozygous missense mutation (A3054C) resulting in a Q981P amino acid substitution. This mutation, which to our knowledge has never before been described, affects the core domain of RAG1, in a region known to contain other amino acid substitutions (R973, K992) found in OS patients $(11,13)$. Finally, P3 presented a homozygous C2633T missense mutation leading to a R841W substitution. This mutation has been observed in OS patients (13). Thus, the RAG1 mutations identified in 3 patients (P1, P3, and P4) are known to result in residual RAG1 activity and are therefore compatible with the presence of both B and T lymphocytes in these patients. RAG1 Q981P is probably also hypomorphic.

\section{Discussion}

We describe here a new immunodeficiency syndrome in 4 patients with recessive hypomorphic $R A G 1$ mutations. The immunological phenotype consists of TCR $\alpha \beta^{+}$lymphopenia, contrasting with markedly high counts of TCR $\gamma \delta^{+} \mathrm{T}$ cells. All T cells were of autologous origin, thereby excluding the possibility of maternofetal engraftment. These unusual features were associated with the early onset of multivisceral and recurrent CMV infection in all patients and with autoimmune cytopenia in all but 1 patient. Occurrence of the multivisceral CMV infection in an nonimmunocompromised infant was considered unlikely because of the associated infectious and persistent autoimmune manifestations, although these findings were not regarded as a definitive exclusion criterion, since coinfection and autoimmunity have been reported in infants with CMV infection (32). However, the persistent detection of low TCR $\alpha \beta$ T cell counts in all 4 patients was considered to be a con- 
A
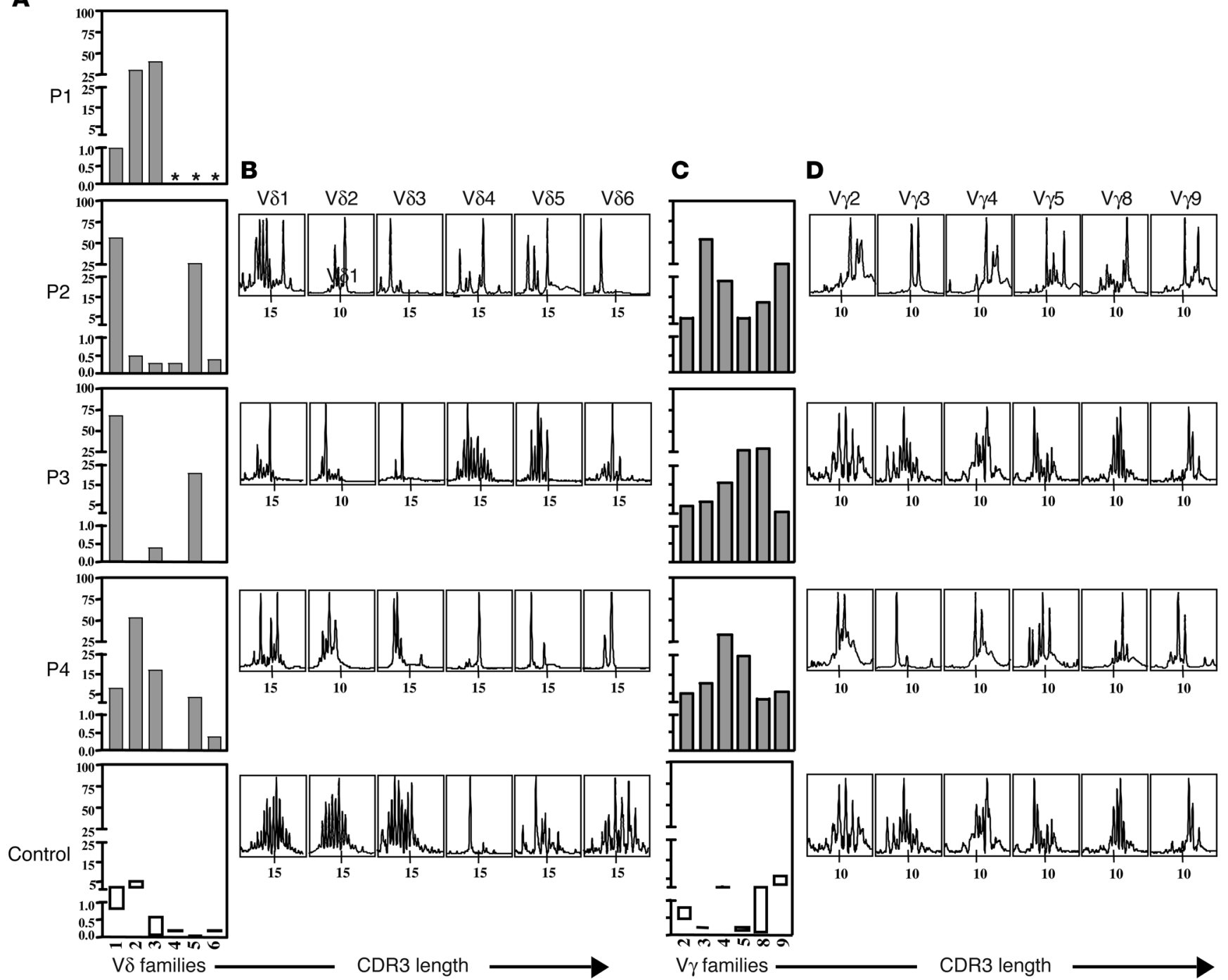

CDR3 length

Figure 2

$\mathrm{TCRV} \gamma$ and $-\mathrm{V} \delta$ usage by patients' T lymphocytes. $\mathrm{V} \delta(\mathbf{A})$ and $\mathrm{V} \gamma(\mathbf{C})$ repertoire was determined either using available specific mAb in immunofluorescence assay in P1 or using real-time PCR analysis in P2, P3, and P4. The $x$ axis indicates V $\delta$ family usage by TCR $\gamma \delta$ cells; the $y$ axis indicates relative frequency (\%) within the TCR $\gamma \delta$ T cells. Controls values shown in rectangles represent mean \pm 2 SDs. Similar results were obtained using available specific mAb in immunofluorescence assay (data not shown). *Not done. Profiles of the fluorescent $\mathrm{V} \delta$-C $\delta(\mathbf{B})$ and $\mathrm{V} \gamma-\mathrm{C} \gamma(\mathrm{D})$ runoff products obtained with mononuclear cells. The $x$ axis indicates CDR3 length.

sequence of a primary immunodeficiency in the absence of HIV infection. Because of the restricted TCR repertoire, a partial T cell developmental defect was envisaged. A V(D)J recombination defect was thus considered. A genome-wide scan found a linkage in the 11 p region where the RAG1/RAG2-encoding genes are located. The causative role of the RAG1 mutations found in these patients was demonstrated in 2 ways: (a) no other known cause of primary immunodeficiency was found, despite thorough immunological investigations, and (b) 3 of the 4 mutations detected in these patients have been identified in patients with an immunodeficiency called Omenn syndrome (mutations found in P1 and P4) or atypical Omenn syndrome (mutation found in P3) (11, 13, 29-31). These mutations have been found to be hypomorphic: del T631 and del 368-369 lead to translation starting from an alternative initiation site, at a second methionine codon downstream from the deletion (31). The missense mutations found in P2 (Q981P) and P3 (R841W) involve amino acids within the minimal core of RAG1, but the mutant proteins nonetheless display residual RAG1 activity. The R841W mutation has been found in OS patients (13), and RAG1 mutations leading to residue changes at positions close to 981 (R973, K992, R975) have also been reported to encode proteins with residual activities in vitro (13).

These findings broaden the spectrum of disease phenotypes observed in patients carrying recessive mutations of the RAG1 and RAG2 genes. Patients with null alleles display a typical T- $\mathrm{B}^{-}$ SCID phenotype, as no productive rearrangement of the TCR or BCR can occur (13). In contrast, 2 other phenotypes have been described for patients carrying hypomorphic mutations. The most frequent - OS - involves the oligoclonal expansion of T cells with a Th2 phenotype, with eosinophilia and hyper-IgE syndrome. Th2 


\section{Table 2}

CDR3 sequences of $V \delta 3$ and $V \gamma 3$ in patient 4

\begin{tabular}{|c|c|c|c|c|}
\hline \multicolumn{3}{|c|}{ Nuclei sequences } & \multirow[t]{2}{*}{$\mathbf{F}$} & \multirow[t]{2}{*}{ L } \\
\hline V $\delta 3$ & & $\mathbf{C \delta}$ & & \\
\hline TGTGCCTGT & I AGCGGGGGATACGGCGATAAACTC & ATCTTTGGAAAAGGAACCCGTGTGACTGTGGAACCA & 1 & 8 \\
\hline TGTGCCTTT & GGGGGGGTCGGTTGGACCGATAAACTC & ATCTTTGGAAAAGGAACCCGTGTGACTGTGGAACCA & 1 & 9 \\
\hline TGTGCCTCA & AAGGCCCACTGGGGGGCGTCCGATAAACTC & ATCTTTGGAAAAGGAACCCGTGTGACTGTGGAACCA & 2 & \\
\hline TGTGCCGTC & CGGGGGATACGTTATGACACCGATAAACTC & ATCTTTGGAAAAGGAACCCGTGTGACTGTGGAACCA & 1 & \\
\hline TGTGCCTGT & T TCGTGGGGGGGGATAGGCCCCGATAAACTC & ATCTTTGGAAAAGGAACCCGTGTGACTGTGGAACCA & 6 & 10 \\
\hline TGTGCCTAC & TGGTACTGGGGGATACCGGACGATAAACTC & ATCTTTGGAAAAGGAACCCGTGTGACTGTGGAACCA & 16 & \\
\hline TGTGCCTTT & TTTCCCTACTGGGGTTTACCCGGGGATAAACTC & ATCTTTGGAAAAGGAACCCGTGTGACTGTGGAACCA & 78 & 11 \\
\hline TGTGCCTCC & TTAGGGGGGACGTACTGGGGGATATCCCATAAACTC & ATCTTTGGAAAAGGAACCCGTGTGACTGTGGAACCA & 5 & \\
\hline TGTGCCTCC & CTGGAGTGGGGGAATCCCTCTTACACCGATAAACTC & ATCTTTGGAAAAGGAACCCGTGTGACTGTGGAACCA & 3 & 12 \\
\hline TGTGCCTTT & GGTTCTCGTACTGGGGGATACGCGAGTACCGATAAACTC & ATCTTTGGAAAAGGAACCCGTGTGACTGTGGAACCA & 1 & 13 \\
\hline TGTGCCTTT & AAACGACGGAGACCCTTCCTAAAGTGGGATACTTACAAACTC & ATCTTTGGAAAAGGAACCCGTGTGACTGTGGAACCA & 1 & 14 \\
\hline TGTGCCTTT & AAGGCGCCCTACCAGTCCTACTGGGGGATCCTTCCTGTTGCCACCGATAAACTC & ATCTTTGGAAAAGGAACCCGTGTGACTGTGGAACCA & 1 & 18 \\
\hline $\mathbf{V}_{\gamma \mathbf{3}}$ & & $\mathbf{C}_{\gamma}$ & & \\
\hline GCCACC & TGGCTCTATTATAAGAAA & CTCTTTGGCAGTGGAACAACA & 46 & \\
\hline GCCACC & TGGGACATCCTGGGGAAA & CTCTTTGGCAGTGGAACAACA & 6 & \\
\hline GCCACC & TGGGACAGGCGTGGGAAA & CTCTTTGGCAGTGGAACAACA & 1 & 6 \\
\hline GCCACC & AGGATTTATTATAAGAAA & CTCTTTGGCAGTGGAACAACA & 1 & \\
\hline GCCACC & CGGCTTTATTATAAGAAA & CTCTTTGGCAGTGGAACAACA & 1 & \\
\hline GCCACC & CGGCTCTATTATAAGAAA & CTCTTTGGCAGTGGAACAACA & 1 & \\
\hline GCCACC & TGGGACATTTATTATAAGAAA & CTCTTTGGCAGTGGAACAACA & 1 & 7 \\
\hline GCCACC & TGGGATGGGTGGCGTTATAAGAAA & CTCTTTGGCAGTGGAACAACA & 1 & \\
\hline GCCACC & TGGGACAGGCGCGGTTATAAGAAA & CTCTTTGGCAGTGGAACAACA & 2 & 8 \\
\hline GCCACC & TGGGACGAGCCGAATTATTATAAGAAA & CTCTTTGGCAGTGGAACAACA & 7 & \\
\hline GCCACC & TGGGACCTCACCAATTATTATAAGAAA & CTCTTTGGCAGTGGAACAACA & 1 & 9 \\
\hline GCCACC & TGGGACCTCACCACTTATTATAAGAAA & CTCTTTGGCAGTGGAACAACA & 2 & \\
\hline
\end{tabular}

$F$, frequency of clones with identical sequences; L, CDR3 length (aa).

$\mathrm{T}$ cells infiltrate the skin, the gastrointestinal tract, and other organs, possibly in relation to autoimmune reactivity caused by defective central tolerance $(11,14,33)$. Residual RAG1/2 activity enables a certain number of precursor cells to rearrange their TCR and BCR genes successfully $(11,29,34)$, this rearrangement being followed by the massive expansion of a few autoaggressive $\mathrm{T}$ cell clones (14). Villa et al. described a third phenotype associated with recessive RAG1 or RAG2 mutations, which they named "atypical SCID/Omenn syndrome" (13). In patients with this syndrome, $\mathrm{T}$ cells are detectable in the periphery, albeit in small numbers, and there are only milder manifestations of immunopathological reactions in the skin or gastrointestinal tract, but most $T$ cells express memory/activation markers. In half of these patients, $B$ cells are also detectable (13).

The RAG1/2 gene mutations causing the OS and atypical SCID/ OS phenotypes have yet to be clearly defined. There is clearly an overlap, because the same mutations have been found in patients with each of these phenotypes (A444V mutation, for example)
(11). A milder phenotype has also been described in a 6-year-old girl presenting a combination of maternal $\mathrm{T}$ cells and autologous functional B cells that produced anti-herpes simplex virus antibody upon infection. This phenotype was associated with 2 mutations of RAG1, one leading to the insertion of a premature stop codon in RAG1 (R897X) and the second being a missense mutation (R559S) in the core region of RAG1 (35). The missense mutant has been shown to display low but detectable levels of recombination activity in a cellular assay (35). Further overlap between a typical $\mathrm{T}^{-} \mathrm{B}^{-}$SCID phenotype and OS, as observed in patients with the same mutations in several families (11), has suggested that the phenotypic consequences of RAG1 or RAG2 mutations depend on (a) the existence of residual $V(D) J$ recombination activity $(11,31,34)$ and (b) other yet-unknown genetic or environmentally determined factors. In this setting, it is tempting to conclude that the phenotype observed in the 4 patients described here results from such an environmental factor. The common feature of all 4 patients is early CMV infection. In all 4 
Table 3

Serum Ig and antibody determinations

\begin{tabular}{|c|c|c|c|c|c|}
\hline & P1 & P2 & P3 & P4 & $\begin{array}{c}\text { Age- } \\
\text { matched } \\
\text { normal } \\
\text { values }\end{array}$ \\
\hline Age at investigation (mo) & 3 & $12-19$ & 13 & 19 & \\
\hline \multicolumn{6}{|l|}{ Igs } \\
\hline $\operatorname{lgG}, \mathrm{mg} / \mathrm{dl}$ & $<200$ & $700-1240$ & $650-1220$ & $743^{A} 4$ & $420-800$ \\
\hline $\lg A, \mathrm{mg} / \mathrm{dl}$ & 7 & $30-120$ & $38-48$ & 0 & $16-68$ \\
\hline $\operatorname{lgM}, \mathrm{mg} / \mathrm{dl}$ & 26 & $240-426$ & $7-85$ & 270 & $50-98$ \\
\hline $\operatorname{lgE}, \mathrm{IU} / \mathrm{ml}$ & ND & 11 & 3.6 & $<4$ & $10-25$ \\
\hline \multicolumn{6}{|l|}{ Antibodies against } \\
\hline Tetanus toxoid (IU/ml) & ND & $<0.06$ & NE & NE & $>0.125$ \\
\hline Polio virus (titer $\times 10^{-1}$ ) & ND & $<10$ & NE & $N E$ & $>40$ \\
\hline । & ND & 20 & NE & NE & $>40$ \\
\hline III & ND & 40 & NE & NE & $>40$ \\
\hline CMV IgG & ND & 11 & 31 & 32 & $<0.9$ \\
\hline CMV IgM & ND & Positive & Negative $\mathrm{P}$ & Positive & \\
\hline
\end{tabular}

AUnder intravenous Ig treatment.

patients, dissemination and recurrence of infection was observed each time antiviral therapy was decreased or stopped, probably reflecting a limited appropriate anti-CMV T cell immunity. The severity of the immunodeficiency was also demonstrated by the severe coinfections observed in 2 patients (Psendomonas aeruginosa sepsis, Pneumocystis jerovici pneumonitis). Thus, prolonged CMV infection in patients with profound (but not complete) inherited $\mathrm{T}$ cell immunodeficiency may have led to the activation and expansion of $\gamma \delta^{+} T$ cell clones. This assumption is based on the known ability of $\gamma \delta^{+} \mathrm{T}$ cells to recognize products from microorganisms directly. The expansion of various $\gamma \delta \mathrm{T}$ cells has been reported to occur in recipients of kidney allografts developing CMV infection (23). In this case, most of the $\mathrm{V} \delta 1^{+}$and $\mathrm{V} \delta 3^{+}$ $\mathrm{T}$ cells detected were activated in vitro by CMV, demonstrating their specificity $(23,25)$. An analysis of junctional diversity in these $\gamma \delta^{+} \mathrm{T}$ cells showed that the size distribution of CDR3 cells was restricted, indicating the probable selective expansion of antigen-specific clones (36). In the 4 patients carrying hypomorphic RAG1 mutations, the diversity of TCR $\gamma$ and $\delta$ CDR3 chain length was found to be fairly restricted. In vitro assay of $\mathrm{CMV}$-infected stromal cell-induced $\gamma \delta \mathrm{T}$ cell proliferation, cytotoxic activity, and TNF- $\alpha$ release (25) did not provide evidence to suggest that $\gamma \delta$ T cells were CMV specific. Nevertheless, the striking similarity between these patients' phenotype and the one of CMV-infected kidney transplant recipients with acquired immunodeficiency strongly suggests that expansion of the population of $\gamma \delta$ TCR T cells is directly related to the persistence of CMV infection $(23,25)$. The predominant location of CMV infection in mucosae (lung and gastrointestinal tract, where mostly Vdelta2 ${ }^{-} \gamma \delta$ T cells are located; ref. 37) could also favor local $\gamma \delta \mathrm{T}$ cell population expansion. Of note, in mice, $V \delta_{1}$ cells were also found to exert a protective effect against CMV infection in the liver (38). The lack of in vitro-detectable reactivity to CMV of patients' TCR $\gamma \delta$ T cells can either reflect usage of inadequate assays or the fact that these $\gamma \delta \mathrm{T}$ cells do not directly react to CMV molecules. It has not been proven that expanded $\gamma \delta \mathrm{T}$ cells found in chronically infected patients after organ transplanta- tion directly recognize CMV molecules, although the $\gamma \delta$ TCR is required for activation (25). $V \delta_{1} \mathrm{~T}$ cells have been shown to be activable by self lipids associated to $\operatorname{CD} 1 \mathrm{c}(39,40)$ or through stress-induced MICA/B MHC class I-related molecules $(41,42)$. Presence of IL-12 produced by dendritic cells has also been shown to be necessary to induce in vitro $\mathrm{V}_{1} \mathrm{~T}$ cell activation (43). It is thus possible that chronic CMV infection, in patients with partial RAG1 deficiency, triggered the expression of stress proteins and/or self lipids leading to the activation of these $\gamma \delta$ T cells. $\mathrm{CMV}$ products might also trigger a superantigen effect, although the diversity of the TCRV $\delta$ and TCRV $\gamma$ families (with restricted CDR3 length distribution) in the patients is consistent with the antigen-induced activation of a particular set of available clones. The CD45RO ${ }^{+} \mathrm{CD} 27^{-} \mathrm{CD} 28^{-}$phenotype of the $\gamma \delta \mathrm{T}$ cells is itself evidence for in vivo activation.

Another striking feature in 3 of these 4 patients was autoimmunity to blood cells (anemia in 3 patients and neutropenia in 1). No such autoimmunity has previously been reported in patients with RAG1/2 mutations, with the exception of 1 patient with mild immunodeficiency who presented both autoimmune thrombocytopenia and neutropenia (35). This may reflect a trend toward the development of antibody-mediated immunity, provided that the disease is not fatal in the first few months of life and that B cells with sufficiently diverse BCRs are present, which is not the case in most patients with RAG1/2 mutations. Nevertheless, it is also possible that the expanded $\gamma \delta \mathrm{T}$ cell clones do also promote autoimmunity, as previously suggested (44). Chronic CMV infections are also known to be frequently associated with autoimmune hemolytic anemia, possibly due to the production of cross-reactive antibodies (45). An antibody production deficiency was also found in these patients. It may be the consequence of defective $T$ cell help and/or reduced $B$ cell repertoire diversity in the setting of a defective $V(D) J$ rearrangement of B cell receptor genes.

In conclusion, this report extends the spectrum of disease phenotypes associated with $R A G 1 / 2$ mutations (Table 4). It strongly suggests that further phenotypic variability will be identified due to the effects of RAG1/2 mutations, genetic background, and infectious environment. This finding may well apply to many other primary immunodeficiency diseases, such as X-linked BTK deficiency, the phenotype of which extends from agammaglobulinemia to a limited deficiency of antibody to polysaccharidic antigens $(46,47)$.

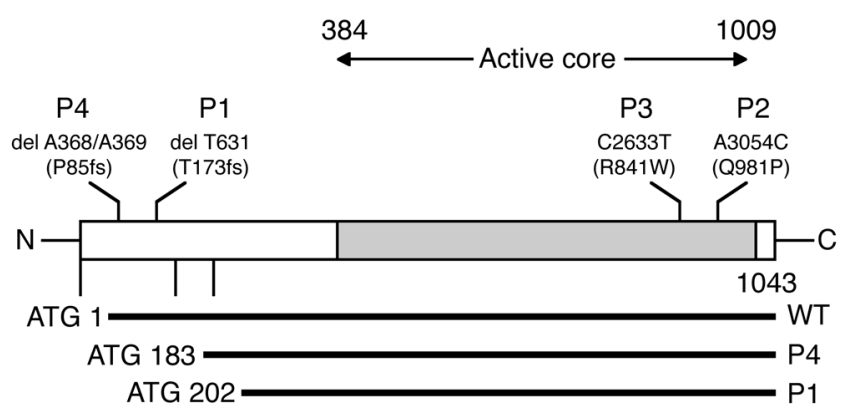

\section{Figure 3}

Schematic representation of the RAG1 gene mutations. Mutations are represented and placed on the overall schematic primary structure of the RAG1 protein. Horizontal bars represent the expected truncated products as compared with the wild-type RAG1 protein. fs, frameshift. 


\section{Table 4}

Spectrum of primary immunodeficiencies associated with RAG1 mutations

\begin{tabular}{|c|c|c|c|c|}
\hline & T-B-SCID & OS & Atypical SCID/OS-mild ID & $\begin{array}{c}\text { ID with } \gamma \delta \text { T cell expansion } \\
\text { and autoimmunity }\end{array}$ \\
\hline T cells & 0 & $\mathrm{~N}, \uparrow$ (oligoclonal) & $\downarrow$ & $\mathrm{N}(\gamma \delta$ oligoclonal) \\
\hline B cells & 0 & $0, \downarrow \downarrow$ & $\downarrow \downarrow$ to $\mathrm{N}$ & $\downarrow$ to $\mathrm{N}$ \\
\hline RAG1 mutation consequences & Null & Hypomorphic & Hypomorphic & Hypomorphic \\
\hline Associated factors & - & $?$ & $?$ & CMV infection \\
\hline
\end{tabular}

ID, immunodeficiency. N, normal; $\uparrow$, increased; $\downarrow$, moderately decreased; $\downarrow \downarrow$, profoundly decreased.

\section{Methods}

\section{Patients}

P1. P1 was the first child born to first-cousin parents of Algerian origin. The child was healthy at birth. At 3 months of age, he was referred to Necker-Enfants Malades Hospital for Pseudomonas aeruginosa sepsis and CMV pneumonitis. CMV was detected in blood (viral load determined by PCR: 39,800 copies/ $\mathrm{ml}$ of blood), in bronchial aspirates, and in urine. The immunodeficiency of this patient was treated by haploidentical $\mathrm{T}$ cell-depleted bone marrow transplantation (BMT), with the mother as a donor, following a conditioning regimen. Acute GVHD, together with reactivation of CMV disease, resulted in the patient's death 150 days after BMT.

P2. P2, a girl born to first-cousin parents of Moroccan origin after an uneventful pregnancy, was referred to Necker-Enfants Malades Hospital at 5 months of age. She had interstitial pneumonitis caused by Pneumocystis carini and CMV. CMV was also found in blood and urine specimens. At 1 year of age, she presented fever, protracted diarrhea, severe autoimmune neutropenia, and hemolytic anemia associated with CMV disease reactivation. The patient was treated with ganciclovir together with G-CSF and steroids. CMV infection was reactivated at 14 months and 18 months (pneumonitis) and treated with a combination of ganciclovir and Foscavir. At 19 months of age, the patient received a haploidentical, $\mathrm{T}$ cell-depleted bone marrow transplant, with her father as the donor, after a conditioning regimen. Liver and gastrointestinal GVHD, with the reactivation of CMV disease and autoimmune anemia, resulted in the patient's death 6 months after BMT.

P3. P3 was a boy born to first-cousins parents of Lebanese origin after an uneventful pregnancy. At the age of 6 months, he presented autoimmune hemolytic anemia, which was treated with steroids. At 10 months of age, he presented pneumonitis associated with hepatosplenomegaly and recurrence of hemolytic anemia. Lung biopsy showed severe bronchiolitis lesions. Pseudomonas aeruginosa, Candida albicans, and CMV grew from bronchoalveolar fluid. CMV was also detected in urine and blood. The child received appropriate anti-infection treatment, including ganciclovir and Foscavir. The patient was referred to Necker-Enfants Malades Hospital at 14 months of age, where he underwent BMT with HLA-identical bone marrow from his $\mathrm{CMV}^{+}$mother, after a conditioning regimen. BMT was complicated by a severe skin, liver, and pulmonary GVHD, associated with CMV disease reactivation and autoimmune anemia, eventually leading to death 14 months after BMT.

P4. P4 was born to related parents of Turkish origin. At 5 months of age, she was referred to St Justine Hospital for severe autoimmune hemolytic anemia requiring treatment with a combination of steroids and rituximab. CMV was detected in urine, saliva, and blood. Appropriate treatment with ganciclovir and Foscavir was initiated. Further reactivation of CMV infection occurred at the ages of 16, 18, and 23 months. The patient underwent haploidentical BMT at 26 months of age, after a conditioning regimen. The donor was her father. Six months after BMT, the patient was doing well, with no significant complications.

The results of complement studies (C3, C4, CH50) were normal, and results of HIV tests were negative for all patients.

Informed consent for the study was obtained from the families in accordance with the Helsinki Declaration.

\section{Immunological investigations}

We used the following mAbs for immunofluorescence analysis: antiCD3: Leu 4 (IgG2a; BD); anti-CD4: Leu 3a (IgG1; BD); anti-CD8: Leu 2a (IgG1; BD); anti-CD19: J4 119 (IgG1; Immunotech, Beckman Coulter); anti-CD56: MY31 (IgG1; BD); anti-TCR $\alpha \beta$ : BMA031 (IgG1; Immunotech, Beckman Coulter); anti-TCR $\gamma \delta$ : IMMU 515 (IgG1; Immunotech, Beckman Coulter); anti-CD45RO: UCHL1 (IgG2a; Immunotech, Beckman Coulter); anti-CD45RA: ALB11 (IgG1; Immunotech, Beckman Coulter); anti-CD31 (IgG2; Immunotech, Beckman Coulter); anti-CD27 (IgG1; Immunotech, Beckman Coulter); anti-CD28 (IgG1; Immunotech, Beckman Coulter). We obtained anti-TCRBV1S1 and -TCRBV1S2 (IgG1), anti-TCRBV2S1 (IgG1), anti-TCRBV3S1 (IgM), anti-TCRVß4 (IgM), anti-TCRBV5S1(IgG2a), anti-TCRBV5S2 (IgG1), anti-TCRBV5S3 (IgG1), anti-TCRBV7S1 (IgG2a), anti-TCRBV8S1 and -TCRBV8S2 (IgG2a), anti-TCRBV9S1 (IgG2a), anti-TCRBV11S1(IgG2a), anti-TCRBV12S2 (IgG2a), anti-TCRBV13S1 (IgG2b), anti-TCRBV13S6 (IgG1), anti-TCRBV14S1 (IgG1), anti-TCRBV16S1 (IgG1), anti-TCRBV17S1 (IgG1), anti-TCRBV18S1 (IgG1), anti-TCRBV20S1 (IgG) and anti-TCRBV21S3 (IgG2a), anti-TCRBV22S1 (IgG1), anti-TCRBV23S1

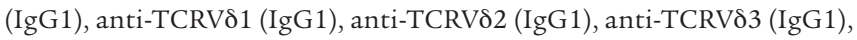
anti-TCRV $\gamma 2 / 3 / 4$ (IgG1), anti-TCRV $3 / 5$ (IgG1), anti-TCRV 8 (IgG1), and anti-TCRV $\gamma 9$ (IgG1) antibodies from Immunotech, Beckman Coulter. Fluorescence staining was carried out with PE- or FITC-conjugated mAbs, on whole blood. Cells were analyzed in a FACScalibur flow cytometer (BD), using Cell Quest software.

Lymphocytes were isolated from patients by Ficoll density gradient centrifugation (Laboratoires Eurobio), and their $\left[{ }^{3} \mathrm{H}\right]$ thymidine uptake was measured in the presence of mitogens (PHA, OKT3) and antigens (tetanus toxoid, final dilution 1:250 [Aventis Merieux]; CMV antigen, final dilution 1:800 [Advanced Biotechnologies Inc.]) (48).

Serum Ig concentrations were determined by nephelometry, and serum antibody concentrations were determined by ELISA (tetanus toxoid, poliovirus, and $\mathrm{CMV}$ ) in patients not receiving intravenous Igs.

\section{RAG1 and RAG2 gene analysis}

DNA was extracted from whole blood by conventional methods, and the RAG1 and RAG2 genes were amplified by PCR and sequenced as previously described (49). The RAG1 gene coding sequence was amplified by PCR from genomic DNA obtained from whole blood using 1F7b: CTGGATCCTTATAAGATACATCAGTGGG; 1092R: CGGCAAGAGGGA- 
CAAT; 1052F: CGGGTCTGCATTCTCAG; 2089R: CTCATCTGCCAGCATAAGG；2013F: TTGCCCACAGCTCTCAGAAT; 2594R: CCTGCCACCTTTTCCTTTC; 2401F: GCGTTCCAACCCTTACC; 3324R: GCCCCATACACAGCAGTA primers and the Expand High Fidelity PCR System (Roche Diagnostics Corp.) according to the manufacturer's recommendations. Nucleotide primers were designed based on the HuRAG1 sequence obtained by Schatz and colleagues (50).

\section{$P C R$ analysis of $V \delta-C \delta$ and $V \beta-C \beta$ junctional diversity}

TCRVB quantitative immunoscope analysis. As previously described (51), cDNA prepared from the P3 PBMCs was amplified with each of the 24 TCRVB family-specific primers together with a TCRBC primer and MGB-TaqMan probe (Applied Biosystems) for TCRBC. Real-time quantitative PCR was carried out on an ABI PRISM 7300 system (Applied Biosystems). PCR products were then subjected to run-off reactions using a nested fluorescent primer specific for the $C \beta$ segment. The fluorescent products were separated and analyzed on a 373A sequencer (Applied Biosystems). The size and intensity of each band were analyzed with Immunoscope software (52). The Gaussian distribution of the different CDR3 lengths was characteristic of normal $V \beta$ repertoire.

$T C R V \gamma \delta$ quantitative immunoscope analysis. As previously described (36), cDNA produced from $0.2 \mathrm{mg}$ of RNA was amplified in a total volume of $25 \mu \mathrm{l}$ with each of the $6 \mathrm{TCRV} \gamma$ and the $6 \mathrm{TCRV} \delta$ primers specific for the TCRV families, in combination with a $C \delta$ or $C \gamma$ primer. Realtime quantitative PCR was carried out on an ABI PRISM 5700 system (Applied Biosystems) and optimized for the SYBR green dye assay. The PCR cycling schedule was as follows: heating for 10 minutes at $95^{\circ} \mathrm{C}$, followed by 40 cycles of 15 seconds at $95^{\circ} \mathrm{C}$ and 1 minute at $60^{\circ} \mathrm{C}$. During PCR, the SYBR green dye binds to double-stranded DNA; the increase in fluorescence is therefore proportional to DNA concentration. PCR products were then subjected to run-off reactions using nested fluorescent primers specific for the $\mathrm{C} \delta$ or $\mathrm{C} \gamma$ segment. The fluorescent products were separated and analyzed on a $373 \mathrm{~A}$ sequencer. The size and intensity of each band were analyzed with Immunoscope software. Fluorescence intensity was plotted in arbitrary units on the $y$ axis, whereas the $x$ axis corresponds to CDR3 length in amino acids.
The $\mathrm{V}$ gene family-specific and constant primers used, oriented 5' to $3^{\prime}$, were as follows: V82: TACCGAGAAAAGGACATCTATGGC; C $\delta$ : TGGGAGAGATGACAATAGCAGGATC; $C \delta$-fam: ACGGATGGTTTGGTATGAGGCTGA; V $\gamma 4$ : CGGAAGCACAAGGAAGAACTTGAGAAT; C $\gamma$ : GAATCGTGTTGCTCTTCTTTTCTTGCC; and $\mathrm{C} \gamma$-fam: ATAGTGGGCTTGGGGGAAACATC.

Sequence analysis of TCR transcripts. PCR of $\mathrm{V} \delta 3-\mathrm{C} \delta$ or $\mathrm{V} \gamma 3-\mathrm{C} \gamma$ was performed on P4 cDNA with $5 \mathrm{U}$ of Pfu polymerase (Stratagene) for 25 cycles. PCR products were then cloned into PCR-blunt II-TOPO vector using the Zero Blunt TOPO PCR Cloning Kit (Invitrogen Corp.). For sequencing purposes, PCR was conducted directly on E. coli colonies with Taq polymerase. Sequencing reactions were performed on these products using $C \delta$ or $\mathrm{C} \gamma$ primers and the ABI PRISM Big Dye Terminator Cycle Sequencing Ready Reaction Kit (Applied Biosystems). These sequencing reactions were run on an ABI PRISM 3730 DNA analyzer. Sequences corresponding to the CDR3 region were extracted and analyzed.

\section{Acknowledgments}

We thank Mark Lathrop from the Centre National de Génotypage (Evry) for scientific advice and Nathalie Lambert, Cécile Dumont, Corinne Jacques, and Chantal Harré (Centre d'Etude des Déficits Immunitaires) for expert technical assistance. This work was supported by an institutional INSERM grant, EUROPOLICY-PID no. PL 006411, and Telethone/AFM grant GAT0203 and by Institut Pasteur.

Received for publication March 28, 2005, and accepted in revised form August 23, 2005.

Jean-Pierre de Villartay and Annick Lim contributed equally to this work.

Address correspondence to: Alain Fischer, Développement Normal et Pathologique du Système Immunitaire, INSERM U429, 149 rue de Sèvres, 75015 Paris, France. Phone: 33-144495071; Fax: 33144495070; E-mail: fischer@necker.fr.
1. Notarangelo, L., et al. 2004. Primary immunodeficiency diseases: an update. J. Allergy Clin. Immunol. 114:677-687.

2. Hirschhorn, R. 1990. Adenosine deaminase deficiency. Immunodefic. Rev. 2:175-198.

3. Leonard, W.J., Noguchi, M., Russell, S.M., and McBride, O.W. 1994. The molecular basis of Xlinked severe combined immunodeficiency: the role of the interleukin- 2 receptor gamma chain as a common gamma chain, gamma c. Immunol. Rev. 138:61-86.

4. Macchi, P., et al. 1995. Mutations of Jak-3 gene in patients with autosomal severe combined immune deficiency (SCID). Nature. 377:65-68.

5. Puel, A., Ziegler, S.F., Buckley, R.H., and Leonard, W.J. 1998. Defective IL7R expression in $\mathrm{T}(-) \mathrm{B}(+) \mathrm{NK}(+)$ severe combined immunodeficiency. Nat. Genet. 20:394-397.

6. Kung, C., et al. 2000. Mutations in the tyrosine phosphatase CD45 gene in a child with severe combined immunodeficiency disease. Nat. Med. 6:343-345.

7. Tchilian, E.Z., et al. 2001. A deletion in the gene encoding the CD45 antigen in a patient with SCID. J. Immunol. 166:1308-1313.

8. Dadi, H.K., Simon, A.J., and Roifman, C.M. 2003 Effect of CD3delta deficiency on maturation of alpha/beta and gamma/delta T-cell lineages in severe combined immunodeficiency. N. Engl. J. Med. 349:1821-1828.
9. de Saint Basile, G., et al. 2004. Severe combined immunodeficiency caused by deficiency in either the $\delta$ or the $\varepsilon$ of CD3. J. Clin. Invest. 114:1512-1517. doi:10.1172/JCI200422588.

10. Schwarz, K., et al. 1996. RAG mutations in human B cell-negative SCID. Science. 274:97-99.

11. Corneo, B., et al. 2001. Identical mutations in RAG1 or RAG2 genes leading to defective $V(D) J$ recombinase activity can cause either T-B-severe combined immune deficiency or Omenn syndrome. Blood. 97:2772-2776

12. Moshous, D., et al. 2001. Artemis, a novel DNA double-strand break repair/V(D)J recombination protein, is mutated in human severe combined immune deficiency. Cell. 105:177-186.

13. Villa, A., et al. 2001. V(D)J recombination defects in lymphocytes due to RAG mutations: severe immunodeficiency with a spectrum of clinical presentations. Blood. 97:81-88.

14. Rieux-Laucat, F., et al. 1998. Highly restricted human $\mathrm{T}$ cell repertoire in peripheral blood and tissue-infiltrating lymphocytes in Omenn's syndrome. J. Clin. Invest. 102:312-321.

15. de Saint-Basile, G., et al. 1991. Restricted heterogeneity of $\mathrm{T}$ lymphocytes in combined immunodeficiency with hypereosinophilia (Omenn's syndrome). J. Clin. Invest. 87:1352-1359.

16. Hinz, T., et al. 1997. Identification of the complete expressed human TCR V gamma repertoire by flow cytometry. Int. Immunol. 9:1065-1072.
17. Janis, E.M., Kaufmann, S.H., Schwartz, R.H., and Pardoll, D.M. 1989. Activation of gamma delta T cells in the primary immune response to Mycobacterium tuberculosis. Science. 244:713-716.

18. Hara, T., et al. 1992. Predominant activation and expansion of $\mathrm{V}$ gamma 9-bearing gamma delta $\mathrm{T}$ cells in vivo as well as in vitro in Salmonella infection. J. Clin. Invest. 90:204-210.

19. Ho, M., Webster, H.K., Tongtawe, P., Pattanapanyasat, K., and Weidanz, W.P. 1990. Increased gamma delta $\mathrm{T}$ cells in acute Plasmodium falciparum malaria. Immunol. Lett. 25:139-141.

20. De Paoli, P., et al. 1990. Gamma delta T cell receptor-bearing lymphocytes during Epstein-Barr virus infection. J. Infect. Dis. 161:1013-1016.

21. Poquet, Y., et al. 1998. Expansion of Vgamma9 Vdelta2 $\mathrm{T}$ cells is triggered by Francisella tularensis-derived phosphoantigens in tularemia but not after tularemia vaccination. Infect. Immun. 66:2107-2114.

22. Sciammas, R., Kodukula, P., Tang, Q., Hendricks, R.L., and Bluestone, J.A. 1997. T cell receptorgamma/delta cells protect mice from herpes simplex virus type 1-induced lethal encephalitis. J. Exp. Med. 185:1969-1975.

23. Dechanet, J., et al. 1999. Major expansion of gammadelta T lymphocytes following cytomegalovirus infection in kidney allograft recipients. J. Infect. Dis. 179:1-8.

24. van Dam, J.G., Damoiseaux, J.G., Christiaans, 
M.H., and Bruggeman, C.A. 2000. Acute primary infection with cytomegalovirus (CMV) in kidney transplant recipients results in the appearance of a phenotypically aberrant CD8+ T cell population. Microbiol. Immunol. 44:1011-1017.

25. Halary, F., et al. 2005. Shared reactivity of Vdelta2(neg) gammadelta $\mathrm{T}$ cells against cytomegalovirus-infected cells and tumor intestinal epithelial cells. J. Exp. Med. 201:1567-1578.

26. Appay, V., et al. 2002. Memory CD8+ T cells vary in differentiation phenotype in different persistent virus infections. Nat. Med. 8:379-385.

27. Lander, E.S., and Botstein, D. 1987. Homozygosity mapping: a way to map human recessive traits with the DNA of inbred children. Science. 236:1567-1570.

28. Kruglyak, L., Daly, M.J., Reeve-Daly, M.P., and Lander, E.S. 1996. Parametric and nonparametric linkage analysis: a unified multipoint approach. Am. J. Hum. Genet. 58:1347-1363.

29. Noordzij, J.G., et al. 2002. The immunophenotypic and immunogenotypic B-cell differentiation arrest in bone marrow of RAG-deficient SCID patients corresponds to residual recombination activities of mutated RAG proteins. Blood. 100:2145-2152.

30. Santagata, S., et al. 2000. N-terminal RAG1 frameshift mutations in Omenn's syndrome: internal methionine usage leads to partial V(D)J recombination activity and reveals a fundamental role in vivo for the N-terminal domains. Proc. Natl. Acad. Sci. U. S. A. 97:14572-14577.

31. Noordzij, J.G., et al. 2000. N-terminal truncated human RAG1 proteins can direct T-cell receptor but not immunoglobulin gene rearrangements. Blood. 96:203-209.

32. Ross, S.A., and Boppana, S.B. 2005. Congenital cytomegalovirus infection: outcome and diagnosis. Semin. Pediatr. Infect. Dis. 16:44-49.
33. Cavadini, P., et al. 2005. AIRE deficiency in thymus of 2 patients with Omenn syndrome. J. Clin. Invest. 115:728-732. doi:10.1172/JCI200523087.

34. Villa, A., et al. 1998. Partial V(D)J recombination activity leads to Omenn syndrome. Cell. 93:885-896.

35. Kumaki, S., et al. 2001. Identification of anti-herpes simplex virus antibody-producing B cells in a patient with an atypical RAG1 immunodeficiency. Blood. 98:1464-1468.

36. Dechanet, J., et al. 1999. Implication of gammadelta $T$ cells in the human immune response to cytomegalovirus. J. Clin. Invest. 103:1437-1449.

37. Falini, B., et al. 1989. Distribution of T cells bearing different forms of the $\mathrm{T}$ cell receptor gamma/ delta in normal and pathological human tissues. J. Immunol. 143:2480-2488.

38. Ninomiya, T., et al. 2000. Vgamma1+ gammadelta $\mathrm{T}$ cells play protective roles at an early phase of murine cytomegalovirus infection through production of interferon-gamma. Immunology. 99:187-194.

39. Groh, V., Steinle, A., Bauer, S., and Spies, T. 1998. Recognition of stress-induced MHC molecules by intestinal epithelial gammadelta $\mathrm{T}$ cells. Science. 279:1737-1740.

40. Spada, F.M., et al. 2000. Self-recognition of CD1 by gamma/delta $T$ cells: implications for innate immunity. J. Exp. Med. 191:937-948.

41. Groh, V., et al. 1999. Broad tumor-associated expression and recognition by tumor-derived gamma delta T cells of MICA and MICB. Proc. Natl. Acad. Sci.U. S. A. 96:6879-6884.

42. Wu, J., Groh, V., and Spies, T. 2002. T cell antigen receptor engagement and specificity in the recognition of stress-inducible MHC class I-related chains by human epithelial gamma delta T cells. J. Immunol. 169:1236-1240.
43. Das, H., Sugita, M., and Brenner, M.B. 2004. Mechanisms of Vdelta1 gammadelta $T$ cell activation by microbial components. J. Immunol. 172:6578-6586.

44. Ware, R.E., and Howard, T.A. 1994. Elevated numbers of gamma-delta (gamma delta+) T lymphocytes in children with immune thrombocytopenic purpura. J. Clin. Immunol. 14:237-247.

45. Murray, J.C., et al. 2001. Infantile cytomegalovirusassociated autoimmune hemolytic anemia. J. Pediatr. Hematol. Oncol. 23:318-320.

46. Wood, P.M., et al. 2001. A mutation in Bruton's tyrosine kinase as a cause of selective anti-polysaccharide antibody deficiency. J. Pediatr. 139:148-151.

47. Lindvall, J.M., et al. 2005. Bruton's tyrosine kinase: cell biology, sequence conservation, mutation spectrum, siRNA modifications, and expression profiling. Immunol. Rev. 203:200-215.

48. Andre-Schmutz, I., et al. 2002. Immune reconstitution without graft-versus-host disease after haemopoietic stem-cell transplantation: a phase $1 / 2$ study. Lancet. 360:130-137.

49. Corneo, B., et al. 2000. Three-dimensional clustering of human RAG2 gene mutations in severe combined immune deficiency. J. Biol. Chem. 275:12672-12675.

50. Schatz, D.G., Oettiger, M.A., and Baltimore, D. 1989. The V(D)J recombination activating gene, RAG-1. Cell. 59:1035-1048.

51. Lim, A., et al. 2002. Combination of MHC-peptide multimer-based $\mathrm{T}$ cell sorting with the Immunoscope permits sensitive ex vivo quantitation and follow-up of human CD8+ T cell immune responses. J. Immunol. Methods. 261:177-194.

52. Pannetier, C., et al. 1993. The sizes of the CDR3 hypervariable regions of the murine T-cell receptor beta chains vary as a function of the recombined germ-line segments. Proc. Natl. Acad. Sci. U. S. A. 90:4319-4323. 